\title{
Three-Cyclic Mono- and Dicationic Compounds as Channel Blockers of NMDA and AMPA Receptors
}

\author{
O. I. Barygin, N. V. Luchkina, and D. B. Tikhonov \\ Sechenov Institute of Evolutionary Physiology and Biochemistry, Russian Academy of Sciences, St.Petersburg; \\ e-mail: denistikhonov2002@yahoo.com
}

DOI: $10.1134 / \mathrm{S} 1990747809030118$

Three-cyclic mono- and dicationic compounds (derivatives of 9-aminoacridine) inhibit NMDA and AMPA subtypes of ionotropic glutamate receptors. The aim of the present study was to compare structureactivity relationships and mechanisms of blockade of NMDA and AMPA receptors by these drugs. The experiments were performed by the whole-cell patchclamp method on neurons isolated from rat brain slices. It was demonstrated that derivatives of 9-aminoacridine, which have flat aromatic moieties, are "foot-inthe-door" blockers of NMDA receptor channels, as well as 9-aminoacridine. Binding of such compounds does not allow closure of the activation gate. Disruption of the flat moiety results in trapping mechanism of the block. Thus, we revealed an important structural determinant of the mechanism of the NMDA receptor channel block. Another important feature of flat aromatic compounds is a steep concentration dependence of their action. Probably, these compounds can form dimers in the channel due to stacking interactions of aromatic rings.

9-aminoacridine and its flat aromatic derivatives also exhibit some peculiarities of action on AMPA receptors. The blocking action of these compounds is voltage-independent suggesting shallow localization of the binding site. Unlike classical voltage-dependent AMPA receptor channel blockers, such as derivatives of adamantine and phenylcyclohexyl, dicationic derivatives of 9-aminoacridine are not more potent than monocationic ones. Thus, flat aromatic derivatives of 9-aminoacridine bind to a specific site in AMPA receptor channels. This opens a way to develop a new family of AMPA receptor antagonists. 